\title{
Optical properties of absorbing and non-absorbing aerosols retrieved by cavity ring down (CRD) spectroscopy
}

\author{
A. Abo Riziq ${ }^{1}$, C. Erlick ${ }^{2}$, E. Dinar ${ }^{1}$, and Y. Rudich ${ }^{1}$ \\ ${ }^{1}$ Department of Environmental Sciences, Weizmann Institute of Science, Rehovot 76100, Israel \\ ${ }^{2}$ Department of Atmospheric Sciences, The Hebrew University of Jerusalem, Jerusalem 91904, Israel
}

Received: 2 November 2006 - Published in Atmos. Chem. Phys. Discuss.: 29 November 2006

Revised: 16 February 2007 - Accepted: 8 March 2007 - Published: 21 March 2007

\begin{abstract}
Application of cavity ring down (CRD) spectrometry for measuring the optical properties of pure and mixed laboratory-generated aerosols is presented. The extinction coefficient $\left(\alpha_{\text {ext }}\right)$, extinction cross section $\left(\sigma_{\text {ext }}\right)$ and extinction efficiency $\left(\mathrm{Q}_{\mathrm{ext}}\right)$ were measured for polystyrene spheres (PSS), ammonium sulphate $\left(\left(\mathrm{NH}_{4}\right)_{2}\left(\mathrm{SO}_{4}\right)\right)$, sodium chloride $(\mathrm{NaCl})$, glutaric acid $(\mathrm{GA})$, and Rhodamine-590 aerosols. The refractive indices of the different aerosols were retrieved by comparing the measured extinction efficiency of each aerosol type to the extinction predicted by Mie theory. Aerosols composed of sodium chloride and glutaric acid in different mixing ratios were used as model for mixed aerosols of two non-absorbing materials, and their extinction and complex refractive index were derived. Aerosols composed of Rhodamine-590 and ammonium sulphate in different mixing ratios were used as model for mixing of absorbing and non-absorbing species, and their optical properties were derived. The refractive indices of the mixed aerosols were also calculated by various optical mixing rules. We found that for non-absorbing mixtures, the linear rule, MaxwellGarnett rule, and extended effective medium approximation (EEMA), give comparable results, with the linear mixing rule giving a slightly better fit than the others. Overall, calculations for the mixed aerosols are not as good as for single component aerosols. For absorbing mixtures, the differences between the refractive indices calculated using the mixing rules and those retrieved by CRD are generally higher.
\end{abstract}

\section{Introduction}

Atmospheric aerosols affect Earth's climate both directly and indirectly (Bates et al., 2006; Bellouin et al., 2005; Kaufman et al., 2002; Koren et al., 2004; Lohmann and Feichter, 2005;

Correspondence to: Y. Rudich

(yinon.rudich@weizmann.ac.il)
Ramanathan et al., 2005, 2001). The direct effect of aerosols on climate is by absorbing and/or scattering the incoming solar radiation and outgoing terrestrial radiation. This interaction strongly modifies Earth's radiation budget and hence the climate on regional and global scales. Much attention has been devoted to purely scattering aerosols, such as sulphate aerosols, mostly due to their "cooling effect". More recently, considerable attention has been directed to absorbing aerosols such as soot (Jacobson, 2001; Koren et al., 2004; Menon et al., 2002), dust (Kaufman et al., 2005; Yu et al., 2006), organics (Kanakidou et al., 2005) and mixed aerosols that contain absorbing species and inclusions. Absorbing aerosols can heat the atmosphere and affect atmospheric circulation (Hansen et al., 2005; Jacobson, 2001; Menon et al., 2002) and cloud formation (i.e., the semi-direct effect) (Koren et al., 2004). There is a growing need to understand and measure atmospheric aerosol optical properties in order to better constrain their direct and semi-direct climatic effects.

The ability of aerosols to interact with radiation is dictated by their optical properties, which depend on their physical and chemical characteristics, and on the wavelength of the incident light. The main parameters in this respect are the scattering and absorption coefficients (or efficiencies). The interaction of radiation with particles by either scattering, absorption, or both, leads to attenuation (or extinction) of the incident light. This attenuation can be expressed as $\alpha_{\text {ext }}=\alpha_{\text {sca }}+\alpha_{\text {abs }}$, where $\alpha_{\text {ext }}$ is the extinction coefficient in units of $\left[\mathrm{L}^{\mathrm{t} 1}\right], \alpha_{\mathrm{sca}}$ is the scattering coefficient, and $\alpha_{\mathrm{abs}}$ is the absorption coefficient. By measuring $\alpha_{\text {ext }}$ and $\alpha_{\text {sca }}$, the single scattering albedo, which is the ratio between the scattered light to the total attenuated light $\left(\varpi_{0}=\alpha_{\mathrm{sca}} /\left(\alpha_{\mathrm{sca}}+\alpha_{\mathrm{abs}}\right)\right)$, can be calculated. The single scattering albedo of particles present in the atmosphere is a key parameter needed in climate models and remote sensing applications. Therefore, accurately measuring the scattering and absorption properties of aerosols is crucial for estimating Earth's energy balance. Methods for calculating the refractive index based on

Published by Copernicus GmbH on behalf of the European Geosciences Union. 
chemical composition are also of importance as they enable the calculation of aerosol radiative properties in climate models.

A number of methods for calculating the radiative properties of aerosols of mixed composition (internal mixtures of different aerosol substances and/or mixtures of aerosol substances with water), are used in climate models. For example, a growth function estimated from measurements or from Mie calculations may be applied to describe the change in scattering coefficient as aerosol water content increases (e.g., Bates et al., 2006). Alternatively, Mie scattering calculations may be employed explicitly during the simulation or in a look-up table fashion, using mixing rules (Erlick, 2006) to calculate the effective refractive indices of the mixture or assuming a core plus shell configuration (Jacobson, 2002). Mixing rules currently in use include: (1) molar refraction and absorption (Born and Wolf, 1999; Jacobson, 2002; Stelson, 1990; Tang, 1997); (2) a volume-weighted linear average of the refractive indices, i.e., the "linear" mixing rule (see, e.g., d'Almeida et al., 1991, their Eq. 6.3); (3) the Maxwell-Garnett rule (see Bohren and Huffman, 1983, Sect. 8.5; Bohren, 1983; Chýlek et al., 1984); and (4) the dynamic effective medium approximation (Chýlek, 2000; Jacobson, 2006). While some of these mixing rules have been tested against experimental data for certain substances with certain volume fractions (see Gosse et al., 1997; Erlick, 2006, and references therein), which rules/models are most appropriate, if at all, remains uncertain.

The molar refraction (absorption) mixing rule assumes that the total molar refraction (absorption) of a mixture is given by the linear average of the molar refraction (absorption) of the components in the mixture weighted by their molar volumes, i.e.,

$$
\begin{aligned}
& R_{\mathrm{tot}}=V_{\mathrm{tot}} \frac{n_{\mathrm{tot}}^{2}-1}{n_{\mathrm{tot}}^{2}+2}=\chi_{1} R_{1}+\chi_{2} R_{2} \quad \text { and } \\
& A_{\mathrm{tot}}=V_{\mathrm{tot}} k_{\mathrm{tot}}=\chi_{1} A_{1}+\chi_{2} A_{2}
\end{aligned}
$$

where $R_{\mathrm{tot}}, V_{\mathrm{tot}}, n_{\mathrm{tot}}, A_{\mathrm{tot}}$, and $k_{\mathrm{tot}}$ are the molar refraction, molar volume, real part of the refractive index, molar absorption, and imaginary part of the refractive index of the mixture, respectively, and $\chi_{i}, R_{i}$, and $A_{i}$ are the molar fraction, molar refraction, and molar absorption of the components, respectively. The molar refraction of the components, molar absorption of the components, and total molar volume are given by:

$$
\begin{aligned}
& R_{i}=\frac{M_{i}}{\rho_{i}} \frac{n_{i}^{2}-1}{n_{i}^{2}+2} \\
& A_{i}=\frac{M_{i}}{\rho_{i}} k_{i} \\
& V_{\text {tot }}=\frac{M_{\text {tot }}}{\rho_{\text {tot }}}
\end{aligned}
$$

where $M$ symbolizes molecular weight and $\rho$ symbolizes density.

The "linear mixing rule" assumes that the total real and imaginary refractive indices of the mixture are given by the linear average of the indices of the components weighted by their volume fractions:

$n_{\text {tot }}=f_{1} n_{1}+f_{2} n_{2}$

$k_{\text {tot }}=f_{1} k_{1}+f_{2} k_{2}$,

where $f_{i}$ is the volume fraction of the components.

In the Maxwell-Garnett mixing rule, one or more of the components (usually the undissolved and/or absorbing components) are deemed "inclusions", while the rest of the components comprise a "homogeneous matrix". The inclusions are assumed to be small (dipoles), spherical, randomly distributed throughout the drop, and dilute, such that the effective dielectric constant of the mixture is given by:

$$
\begin{aligned}
\varepsilon_{\text {tot }} & =\varepsilon_{\text {matrix }}+\frac{3 f_{\text {incl }} \varepsilon_{\text {matrix }}\left(\varepsilon_{\text {incl }}-\varepsilon_{\text {matrix }}\right)}{\varepsilon_{\text {incl }}+2 \varepsilon_{\text {matrix }}-f_{\text {incl }}\left(\varepsilon_{\text {incl }}-\varepsilon_{\text {matrix }}\right)} \\
n_{\text {tot }} & =\left(\varepsilon_{\text {tot }}\right)^{1 / 2}
\end{aligned}
$$

where $\varepsilon_{\text {tot }}, \varepsilon_{\text {incl }}$, and $\varepsilon_{\text {matrix }}$ are the complex dielectric constants of the mixture, the inclusions, and the matrix, respectively, $f_{\text {incl }}$ is the volume fraction of the inclusions, and $n_{\text {tot }}$ is the complex refractive index of the mixture.

The dynamic effective medium approximation is an example of a higher order or extended effective medium approximation as compared to the Maxwell-Garnett mixing rule, where the inclusions are allowed higher order effects than the electric dipole. The size or size distribution of the inclusions themselves must be specified.

Cavity Ring Down (CRD) spectroscopy has been recently introduced for measuring extinction coefficients of laboratory and field aerosols. Sappy et al. (1998) pioneered the use of CRD for detecting ambient particles non-resonantly at $532 \mathrm{~nm}$ and $355 \mathrm{~nm}$. Vander Wal and Ticich (1999) used pulsed CRD to study the absorption of soot produced from methane-air flame and to calibrate laser induced incandescence measurements, which are widely used to measure soot volume fraction. Smith and Atkinson (2001) performed simultaneous measurements of extinction by ambient aerosols at $532 \mathrm{~nm}$ and $1064 \mathrm{~nm}$. Using simultaneous measurements at $510.6 \mathrm{~nm}$ and $578.2 \mathrm{~nm}$, Thompson et al. (2002) monitored the change in atmospheric optical extinction coefficient during a wildfire and during a local fireworks event. Strawa et al. (2003) were the first to use continuous wave cavity ring down (CW-CRD) for aerosol studies. Using diode lasers at wavelengths of $690 \mathrm{~nm}$ and $1550 \mathrm{~nm}$, they measured a minimum extinction coefficient for both wavelengths of about $1.5 \times 10^{-8} \mathrm{~cm}^{-1}$ (a better sensitivity could be achieved with higher reflectivity mirrors). By placing a scattering detector at $90^{\circ}$ to the cavity, they measured the scattering coefficient in addition to the extinction coefficient and directly extracted the single scattering albedo $(\varpi)$. Bulatov et al. (2002) used a pulsed dye laser at $620 \mathrm{~nm}$ to study laboratory-generated non-absorbing $\mathrm{NaCl}$ and $\mathrm{CuCl}_{2} \cdot \mathrm{H}_{2} \mathrm{O}$ aerosols. The measured extinction coefficients were compared to Mie scattering calculations. Bulatov et al. (2006) also measured the extinction coefficient of size selected Rhodamine 640 aerosols (a strongly absorbing dye at $615 \mathrm{~nm}$ ). 
This was the first use of CRD to measure optical properties of absorbing organic aerosols (other than soot). Pettersson et al. (2004) demonstrated the use of pulsed laser $(532 \mathrm{~nm})$ CRD to study polystyrene spheres (PSS) and dioctyl sebacate (DOS) aerosols. Their measurements show good quantitative agreement with Mie calculations in the scattering cross section and refractive index. Recently, Lack et al. (2006) applied CRD to derive aerosol extinction coefficient of absorbing PSS aerosols and to calibrate a photoacustic spectroscopy measurements of the absorption coefficient for these aerosols. Moosmuller et al. (2005) used CRD to measure very low extinction in the atmosphere and laboratory environments.

Cavity ring down spectroscopy was developed by O'Keefe and Deacon (1988). Typically, it consists of two highly reflective plano-concave mirrors set opposite to one another. The placement of the mirrors is dependent on the cavity stability conditions. A pulsed or continuous laser beam is coupled into the cavity from one side and performs multiple reflections inside the cavity. A photomultiplier (PMT) is placed at the other side of the cavity and measures the exponential decay of the emerging light intensity. The intensity $(I)$ decay is a result of losses inside the cavity and due to the mirrors:

$I=I_{o} e^{\left[\frac{-\tau}{\tau_{0}}\right]}$

The time constant for an empty cavity, $\tau_{0}$, is:

$\tau_{0}=L / C(1-R)$

where $L$ is the length of the cavity (distance between the two mirrors), $C$ is the speed of light, and $R$ is the reflectivity of the mirrors. This equation depicts the dependence of the ring down time on the cavity length and the mirror reflectivity. When the cavity is filled with an absorbing or scattering medium, the molecules or particles further reduce the intensity on each pass. This process results in a ring down trace with a shorter time constant due to additional terms in the ring down expression, and the time constant is described by:

$\tau=L / C\left(1-R+\alpha_{\mathrm{ext}} d\right)$

where $\alpha_{\text {ext }}$ is the extinction coefficient of the molecules or particles inside the cavity, and $d$ is the actual distance in the cavity filled with the absorbing molecule. The extinction coefficient can be extracted from the difference between the time constant of the empty and the filled cavity:

$\alpha_{\mathrm{ext}}=\frac{L}{C d}\left[\frac{1}{\tau}-\frac{1}{\tau_{0}}\right]$

The extinction coefficient $\left(\alpha_{\text {ext }}\right)$ of homogeneous spheres (aerosols) is described by:

$\alpha_{\text {ext }}=N \sigma_{\text {ext }}=\frac{1}{4} \pi N D^{2} Q_{\text {ext }}$

where $\sigma_{\text {ext }}$ is the extinction cross section, $Q_{\text {ext }}$ is the extinction efficiency of the particles which is the ratio of Beer's law extinction cross section to the geometric area of the particle and is dimensionless, $N$ is the particle number density, and $D$ is the particle diameter. By selecting a monodisperse aerosol population and measuring the particle number density $(N)$, the extinction efficiency $\left(Q_{\text {ext }}\right)$ can be determined. For a fixed wavelength, $Q_{\text {ext }}$ can be measured as a function of the size parameter by performing measurements on a series of monodisperse particles of different sizes. The size parameter, $x$, is the ratio of the particle size $(D)$ to the laser's wavelength $(\lambda)$ and is given by $(x=\pi D / \lambda)$. Having $Q_{\text {ext }}$ as a function of size parameter enables a retrieval of the particle refractive index.

In this study we present the use of cavity ring down (CRD) for determining the extinction efficiency and complex refractive index of pure and mixed aerosols. Specifically, we focus on mixtures of organic and inorganic components, as $30 \%$ to over $80 \%$ of the aerosol mass in the free troposphere contains carbonaceous material, most of it probably organics (Murphy, 2006). For validation of the new setup, we measure $Q_{\text {ext }}$ as a function of size parameter of polystyrene spheres (PSS) and ammonium sulphate (AS, $\left.\left(\mathrm{NH}_{4}\right)_{2} \mathrm{SO}_{4}\right)$ aerosols, both with well-known refractive indices. Then we use the same setup to retrieve the refractive indices of sodium chloride $(\mathrm{NaCl})$, glutaric acid (GA), and Rhodamine-590 aerosols, as pure component aerosols and in mixtures with one another, the mixtures allowing us to test the appropriateness of the theoretical mixing rules.

\section{Experimental}

\subsection{Aerosol generation and classification}

Aqueous solutions (20-500 $\mathrm{mg} \mathrm{L}^{-1}$ ) of the compounds of interest are nebulized using a TSI constant output atomizer (TSI-3076, 25 psi, 2.36 standard liters per minute (SLM) flow), with dry particle-free pure nitrogen, generating a polydisperse distribution of droplets. The mean diameter of the droplets depends on the concentration of the solution. The aerosol flow enters a $3 \mathrm{~L}$ conditioning bulb before entering two silica gel column dryers, resulting in a flow with relative humidity $(\mathrm{RH})<3 \%$. The dry polydisperse aerosol passed through a neutralizer (TSI 3012A) to obtain an equilibrium charge distribution on the particles. A size selected monodisperse aerosol is generated with an electrostatic classifier (TSI Differential Mobility Analyzer (DMA)) operating with 5 SLM dry $(\mathrm{RH}<3 \%)$ clean nitrogen sheath flow and fixed at an applied voltage. The size-selected monodisperse aerosol flow is directed through a dilution apparatus for precise control of particle number concentration. The sample flow (1.2 SLM) is then directed to the CRD cell.

\subsection{Cavity ring down system (CRD)}

The CRD setup is shown in Fig. 1. Briefly, it consists of two highly reflective concave mirrors (curvature radii of $1 \mathrm{~m}$ and 


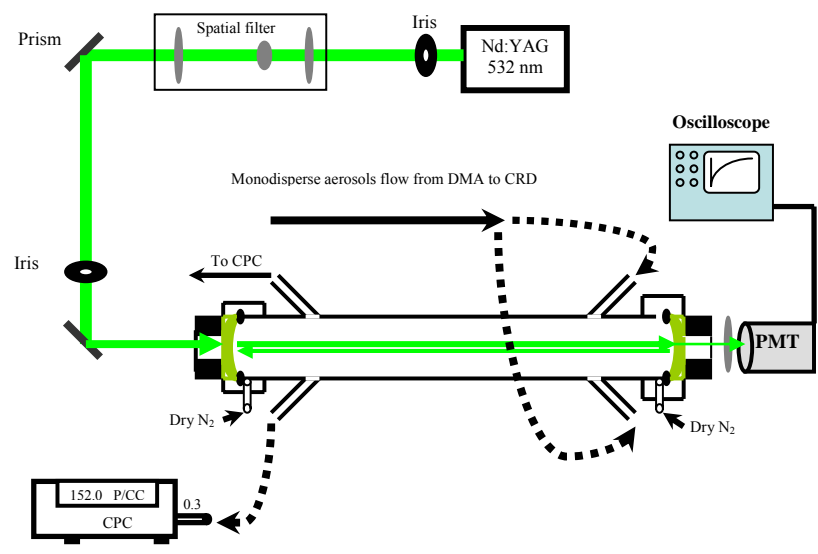

Fig. 1. Schematic representation of the cavity ring down setup for aerosols measurements.

a reflectivity of $99.995 \%$ at $532 \mathrm{~nm}$, Los Gatos, USA). The mirrors are mounted at the two sides of a $90 \mathrm{~cm} \mathrm{3/3"} \mathrm{stain-}$ less steel tube. A small purge flow of dry particle-free nitrogen $(0.05 \mathrm{SLM})$ is introduced in front of each mirror to prevent mirror contamination by deposition of aerosols. The aerosol flow enters the CRD cell through four tubes at $45^{\circ}$. This is designed to ensure good mixing and even concentration of the particles inside the cavity. The flow in each line is $0.3 \mathrm{SLM}$, and the total flow inside the cavity is $1.2 \mathrm{SLM}$. The particles exit the cavity in a similar setup, and their concentration is determined by a condensation particle counter (CPC, TSI 3022A). The length of the cavity occupied by particles during the flow is about $68 \mathrm{~cm}$. To ensure that particle losses are negligible, we measured the particle number density after the DMA and at the exit of the CRD cell. In both cases, the particle number density was almost identical (>98\%) for all particles sizes, suggesting minimal loses in the CRD cell and tubing.

The second harmonic $(532 \mathrm{~nm})$ of a pulsed Nd:YAG laser (Quanta-Ray GCR-100, $10 \mathrm{~Hz}, 7 \mathrm{~ns}$ ) is introduced to the CRD through a spatial filter consisting of two lenses with focal length of $5 \mathrm{~cm}$ and $10 \mathrm{~cm}$ and a $100 \mu$ m-pinhole between the lenses. The beam diameter in front of the cavity is about $1 \mathrm{~mm}$, with energy of about $50 \mu \mathrm{J}$. The intensity of the beam emerging from the CRD cell is measured with a photomultiplier (Hamamatsu H6780-02). The photomultiplier signal is fed into a digital storage oscilloscope (LeCroy, model 9361, $300 \mathrm{MHz}$ ), which is triggered simultaneously with the laser pulse. The digitized data is transferred and stored in a personal computer using a LabVIEW program.

Determining an accurate decay time is critical for precise measurements of the extinction coefficient of the aerosols. Transverse modes inside the cavity lead to non-exponential decay which leads to inaccurate determination of the decay time. Two effects of transverse modes are commonly observed in cavity ring down spectroscopy (Scherer et al., 1996, 1997). The first modulates the decay as a result of multiple

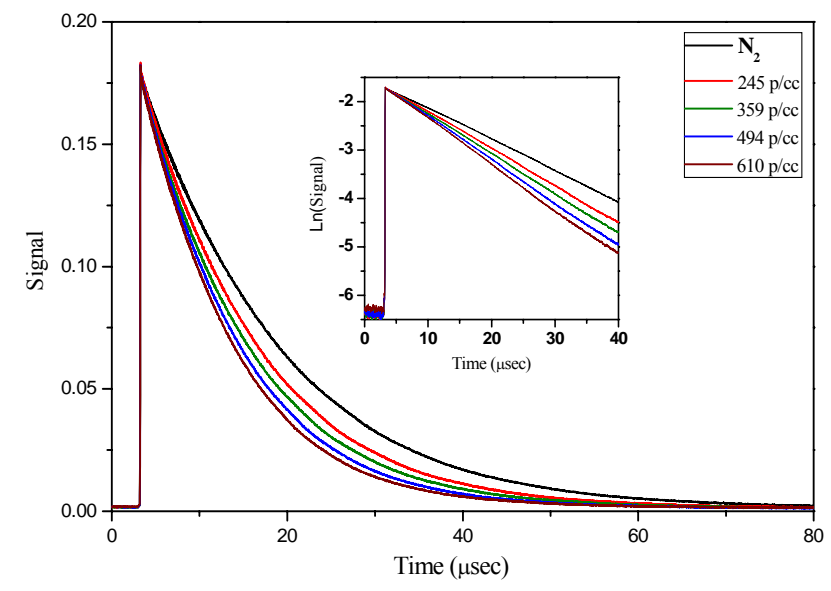

Fig. 2. Experimental decay curves obtained for different concentrations of $400 \mathrm{~nm}$ ammonium sulfate inside the cavity. The slowest decay is obtained for the empty cavity. Each curve is an average of 400 laser shots. The insert pattern shows the natural logarithm of the decay signal as a function of time demonstrating a linear behavior, as expected.

modes which form different optical paths inside the cavity. To overcome this issue we use a spatial filter mode matching using a telescope with a $100 \mu \mathrm{m}$ pinhole between the lenses as described above. The second effect is caused by variations in the quantum efficiency at the detector surface as the laser beam impinges on it. This was overcome by tight focusing of the laser beam on a small surface of the detector, as shown in Fig. 1.

The inset in Fig. 2 shows typical exponential decay curves (on a log scale) in this case of $400 \mathrm{~nm}$ AS particles at different concentrations. The slowest decay is measured when the cavity is filled with a continuous flow of particle-free dry nitrogen resulting with a decay time of $16 \mu \mathrm{s}$. The decay time becomes shorter with introduction of the AS aerosol. Shorter decay times are measured when higher particle concentrations are present in the cavity. The inset in Fig. 2 shows the exponential decays on a log scale. The decay times are extracted from the slope of such lines.

The ability to measure precisely minimal differences in ring down times between an empty cavity $\left(\tau_{o}\right)$ and a cavity filled with aerosols $(\tau)$ provides a good estimate of the maximum sensitivity. To determine the minimum detectable extinction coefficient we use the following definition for the detection limit (Brown et al., 2002):

$\alpha_{\min }=\frac{L}{C \times d} \frac{\Delta \tau_{\min }}{\tau_{o}^{2}}$

where $\Delta \tau_{\min }$.is the standard deviation of the decay time for 400 shots which was obtained for a cavity filled with only dry nitrogen. In the experiments presented here, $\tau_{o}=16 \mu \mathrm{s}\left(\tau_{o}\right.$, is the decay time for cavity filled only with dry nitrogen) and $\Delta \tau_{\min }$ is about $0.02 \mu$ s for an average of a 400 laser shots 


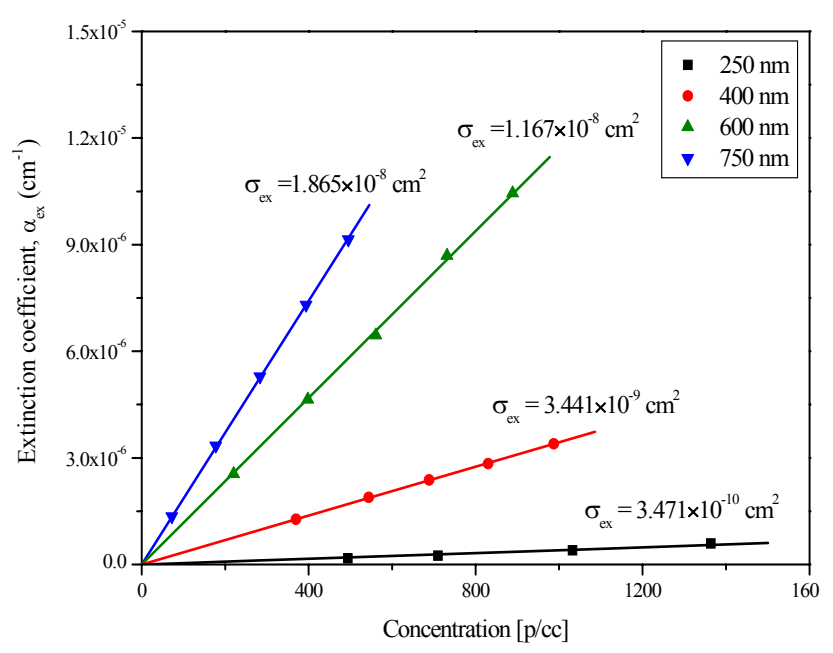

Fig. 3. The extinction coefficient $\left(\alpha_{\text {ext }}\right)$ measured as a function of particle number density of ammonium sulphate at different sizes $(250,400,600$ and $750 \mathrm{~nm})$. The number density was controlled using a dilution apparatus. The extinction cross section $\left(\sigma_{\text {ext }}\right)$ was extracted from a linear fit for each size and is indicated on top of each line.

operating at $10 \mathrm{~Hz}$. This results in a minimum detectable extinction coefficient of $3.77 \times 10^{-9} \mathrm{~cm}^{-1}$. It should be noted that this sensitivity is calculated for a cavity filled with a homogenous distribution of nitrogen; however, in the case of measuring particles such as aerosols, the fluctuation of aerosol concentration inside the cavity would lead to larger fluctuations in decay time and in turn to lower sensitivity. A statistical treatment needs to be performed in the case of aerosols. Since such a treatment is not in the scope of this article, we refer the reader to the detailed treatment by Pettersson et al. (2004), who found that the contribution of fluctuations in aerosol concentration inside the cavity to the total sensitivity is very small leading to sensitivity of the same order as the instrument limit.

\subsection{Retrieval and mixing rule methods}

The retrieval algorithm for single component particles compares the measured extinction efficiency as a function of size parameter with the extinction efficiency calculated using the Mie scattering subroutine for homogeneous spheres by Bohren and Huffman (1983, Appendix A), while simultaneously varying the real and imaginary refractive indices of the particles. It finds the set of refractive indices by minimizing the "merit function" (similar to a variance) $\chi^{2} / N^{2}$, where $\chi^{2}$ is

$\chi^{2}=\sum_{i=1}^{N} \frac{\left(Q_{\text {ext measured }}-Q_{\text {ext calculated }}\right)_{i}^{2}}{\varepsilon_{i}^{2}}$

$N$ is the number of particle sizes, and $\varepsilon$ is the estimated error in the measurement (taken as the standard deviation of

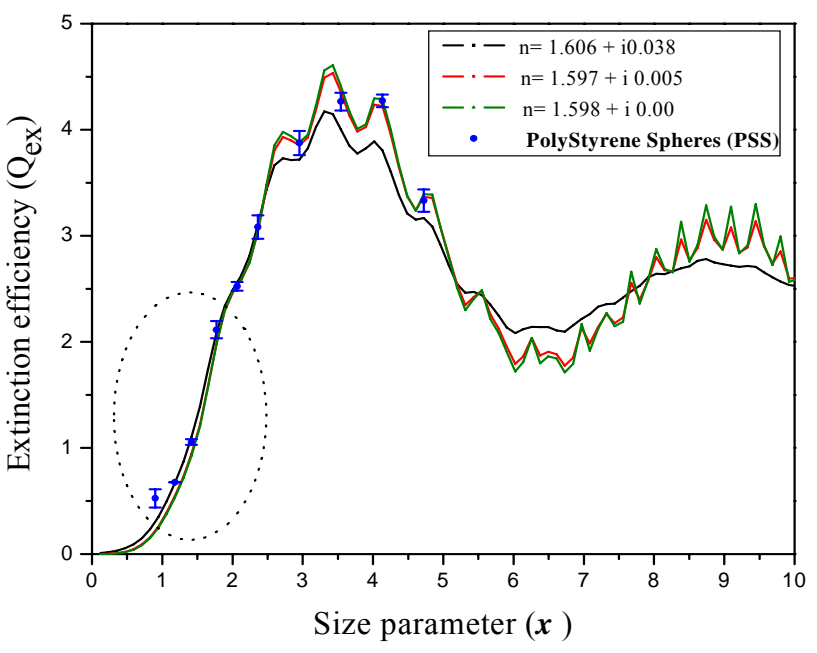

Fig. 4. The extinction efficiency ( $\left.Q_{\text {ext }}\right)$ as a function of size parameter $(x)$ of polystyrene spheres (PSS). The solid curves represent the Mie fit: $n=1.606+i 0.038$ was obtained by fit all the experimental data points, while $\mathrm{n}=1.597+\mathrm{i} 0.005$ was obtained by fit only a subset of sizes starting from $350 \mathrm{~nm}$ (the excluded sizes are enclosed by the dotted circle). The green curve $(n=1.598+i 0.00)$ is from Petterson et al. (2004).

repeated measurements of the same particle size but for different concentrations) (Press et al., 1992, 1992, Eq. 15.5.5). The algorithm does not require an initial guess for the real and imaginary parts of the refractive index. Rather it scans through all possible physical values of the indices and progressively increases the resolution of the search until it finds the absolute minimum in the merit function within the desired precision.

For mixtures of two components, the measured extinction efficiency of the mixture is compared with the extinction efficiency calculated using the mixing rules outlined in Sect. 1, namely, (1) molar refraction and absorption; (2) the volume-weighted linear mixing rule; (3) the MaxwellGarnett rule; and (4) an extended effective medium approximation (EEMA) similar to the dynamic effective medium approximation (Eq. 15 Sihvola and Sharma, 1999), where the effective refractive indices estimated using the mixing rules are input to the Mie scattering subroutine for homogeneous spheres by Bohren and Huffman (1983, Appendix A). The measured extinction efficiency for the mixture is also compared with the extinction efficiency calculated using (5) the core plus shell model, where the refractive indices of the individual components comprising the mixture are input to a layered sphere Mie scattering subroutine (Bohren and Huffman, 1983, Appendix B; coded in Matlab by C. Maetzler, 2004). The merit function for mixtures is defined in the same fashion as for single component particles, and the mixing rule or model with the smallest merit function is deemed the best match. 


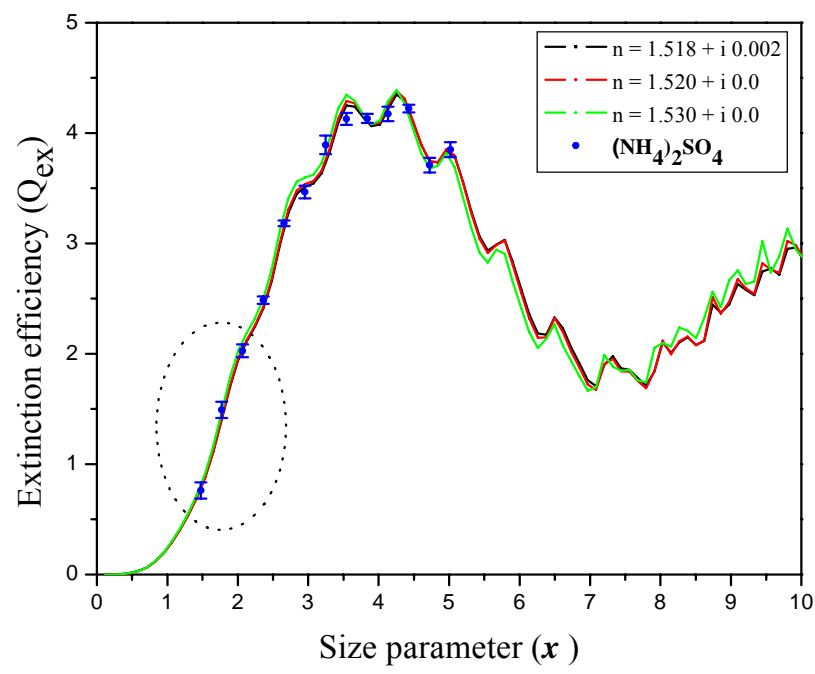

Fig. 5. The extinction efficiency $\left(Q_{\text {ext }}\right)$ as a function of size parameter $(x)$ of ammonium sulphate. The solid curves represent Mie fit:. $\mathrm{n}=1.518+\mathrm{i} 0.002$ was derived from using all the experimental data points, while $\mathrm{n}=1.520+\mathrm{i} 0.00$ was obtained by fit a subset starting from $350 \mathrm{~nm}$. (The excluded sizes are enclosed by the dotted circle.) The green curve $(\mathrm{n}=1.530+\mathrm{i} 0.00)$ is from Petterson et al. (2004).

\section{Results and discussion}

\subsection{Extinction cross section measurements}

In addition to the extinction coefficient, the particle extinction cross section $\left(\sigma_{\text {ext }}\right)$ can be determined by measuring $\alpha_{\text {ext }}$ for different particle concentrations $(N)$, and using the relationship $\alpha_{\text {ext }}=\sigma_{\text {ext }} \times N$. The extinction coefficient as a function of the particle concentration of ammonium sulphate for four different sizes $(250 \mathrm{~nm}, 400 \mathrm{~nm}, 600 \mathrm{~nm}$, and $750 \mathrm{~nm})$, and the corresponding $\sigma_{\text {ext }}$ determined for each size are shown in Fig. 3. It is seen that the extinction increases linearly with particle concentration, as expected.

\subsection{Polystyrene spheres and ammonium sulphate $\left(\mathrm{NH}_{4}\right)_{2} \mathrm{SO}_{4}$}

To test the performance of the new CRD system, we measured the optical properties of polystyrene spheres (PSS) and ammonium sulphate (AS), both with well-known indices of refraction (Lack et al., 2006; Pettersson et al., 2004). We measured the extinction efficiency of 10 different sizes of commercially available PSS (Duke scientific corporation, USA). The extinction efficiency ( $\left.Q_{\text {ext }}\right)$ of these particles as a function of the size parameter (blue squares) is depicted in Fig. 4. The standard deviation of the extinction efficiency $\left(\Delta Q_{\text {ext }}\right)$ is calculated by measuring the extinction efficiency of the same size at different concentrations. The concentration of the particles ranges from 150 to 2500 particles $\mathrm{cm}^{-3}$. To retrieve the index of refraction and to get best fit of Mie theory with the experimental data for $Q_{\text {ext }}$, we used the Mie scattering subroutine described above for two different size ranges, one using all the sizes we measured, and the other using a subset of sizes starting from $350 \mathrm{~nm}$. Three different fitting curves are shown in Fig. 4 in addition to the experimental data obtained for the ten different PSS sizes. The black curve is obtained by a Mie fit for all measured particles sizes, yielding an index of refraction of $\mathrm{n}=1.606+\mathrm{i} 0.038$ with merit function $\left(\chi^{2} / N^{2}\right)$ value of 0.91 . The red curve is obtained for a subset of sizes starting from $350 \mathrm{~nm}$ (excluding the first four points within by the dotted circle), yielding an index of refraction of $\mathrm{n}=1.597+\mathrm{i} 0.005$ with $\chi^{2} / N^{2}=0.06$, substantially lower than the $\chi^{2} / N^{2}$ obtained using all sizes. The green curve is obtained by using $n=1.598+\mathrm{i} 0$, a value reported by Pettersson et al. (2004) for the refractive index of PSS. Our results are in close agreement with the refractive index given by Pettersson et al. (2004) for PSS. However, by using a subset in which the small sizes are excluded we clearly improve our fitting by minimizing the merit function. Note that $\mathrm{n}=1.600+\mathrm{i} 0.000$ (not shown) also provided a low merit value (0.10), so that we cannot rule out an imaginary refractive index of zero as a possibility for the PSS spheres we measured within the precision of our retrieval scheme. It is difficult to determine low imaginary refractive indices with better precision from retrieval schemes in general (see Bohren and Clothiaux, 2006, pp. 163-165).

The extinction efficiency of ammonium sulphate as a function of size parameter for particles sizes between $250 \mathrm{~nm}$ and $850 \mathrm{~nm}$ is shown in Fig. 5. Similar to PSS, we first fit the data for all sizes and then for only a subset of sizes starting from $350 \mathrm{~nm}$. For all the sizes, we obtain $\mathrm{n}=1.518+\mathrm{i} 0.002$ and $\chi^{2} / N^{2}=2.49$ (black curve). For the subset of sizes, we obtain $\mathrm{n}=1.52+0 \mathrm{i}$ and $\chi^{2} / N^{2}=0.14$ (green curve). The red curve is obtained with a refractive index of $n=1.53+\mathrm{i} 0.0$, which is reported to be the index of refraction of the AS (Pettersson et al., 2004). The refractive index of salt aerosols (such as AS) strongly depends on the relative humidity and the crystal structure. In our experiments, the relative humidity is below 3\% (dry aerosols). Therefore, the index of refraction obtained from our measurement should be compared to other studies in which the index of refraction was determined in dry conditions. The index of refraction of dry AS crystals reported for three coordinate axes (orthorhombic crystal structure) are $\mathrm{n}_{\alpha}=1.520, \mathrm{n}_{\beta}=1.523$, and $\mathrm{n}_{\gamma}=1.533$, respectively (Lide, 1997). Our retrieved index of $n=1.52+\mathrm{i} 0.0$ is consistent with that for the third axis, but slightly lower than the other two axes.

For both ammonium sulphate and PSS, excluding the smallest particles slightly improves the fit. A possible reason for this behavior could be the presence of larger multiplycharged particles that would have the same mobility as singly charged particles. In the CRD spectrometer, these multiplycharged particles contribute strongly to the decay time and result in higher extinction efficiency. The multiple charge effect could be reduced by using very dilute solution in the atomizer that shifts the mean diameter in the distribution to 
smaller sizes which reduces the number of multiply charged large particles.

3.3 Sodium chloride $(\mathrm{NaCl})$, glutaric acid and their mixtures

Aerosols in atmosphere are more complex than laboratorygenerated pure aerosols. Typically they are composed of mixtures of organic and inorganic molecules that can be arranged in different ways, such as homogeneous mixtures or as coated particles. Urban and pollution aerosols contain both organic and inorganic components (Murphy et al., 2006), while dust or sea salt particles are often coated by condensed organic and inorganic (Falkovich et al., 2004; Maria et al., 2004; Posfai et al., 1998; Russell et al., 2002; Tervahattu et al., 2002). Therefore, exploring the optical properties of mixed particles is important for exploring the optical properties of atmospheric aerosols. To do so, we studied the optical properties of mixed $\mathrm{NaCl}$ and $\mathrm{GA}$ particles, which are common components of sea salt particles. First we measured the optical properties of pure $\mathrm{NaCl}$ solution and $\mathrm{GA}$ aerosols. Then we measured the optical properties of mixtures of these two components prepared with a known molar ratio $(1: 1,2: 1)$. We assume that the aerosols generated from these solutions are homogenous and that the molar ratio of the solution is maintained in the aerosol, because both compounds dissolve very well in water. This assumption is later verified by calculations of the refractive index.

The extinction efficiency as a function of size parameter for particles of $\mathrm{NaCl}, \mathrm{GA}$, and particles generated from mixtures with molar ratios of $\mathrm{NaCl}$ and GA of 1:1 and 2:1, respectively, are shown in Fig. 6. The standard deviation of the measurements is also shown. The solid lines represent Mie fitting curves obtained using the same refractive index retrieval algorithm described in Sect. 2.3.

A summary of the retrieval results for the mixed particles are given in Table 1. In these experiments, we used very dilute solutions $\left(20-50 \mathrm{mg} \mathrm{L}^{-1}\right)$ for the small particle sizes $(100-300 \mathrm{~nm})$. This clearly improves the fit for all sizes, as is evident from the small differences in the merit functions $\left(\chi^{2} / N^{2}\right)$ between the retrievals for all the sizes and for the subset sizes starting from $350 \mathrm{~nm}$. For example, the retrieval for pure GA aerosols using all sizes yields $n=1.41+\mathrm{i} 0.0$, with $\chi^{2} / N^{2}=0.10$, while the retrieval for pure GA using the subset of sizes yields $\mathrm{n}=1.41+\mathrm{i} 0.0$, with $\chi^{2} / N^{2}=0.13$.

\subsection{Calculations using mixing rules}

As stated in Sect. 1, optical properties of aerosols in the atmosphere are often calculated using various mixing rules and models. The underlying assumption for these mixing rules is that it is possible to calculate the complex refractive index of complex particles through the knowledge of the properties (density, molecular weight, refractive index) of the individual constituents, and the way in which they are mixed.

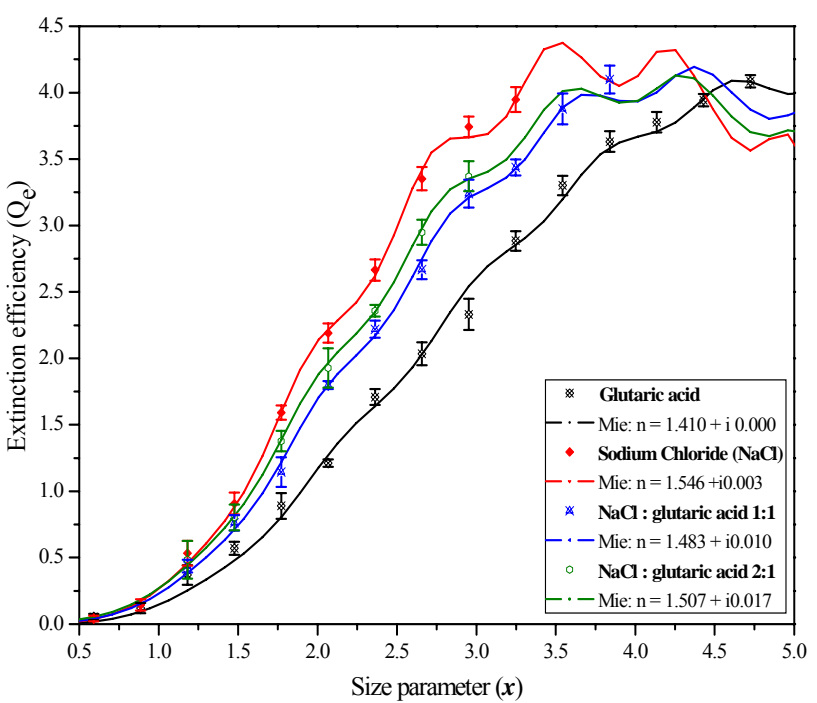

Fig. 6. Extinction efficiency $\left(Q_{\text {ext }}\right)$ as a function of size parameter $(x)$ obtained for sodium chloride, glutaric acid, and the mixtures of $\mathrm{NaCl}$ and glutaric acid with molar ratios 1:1 and 2:1 respectively. The solid curves are the result of the Mie fit to the experimental points.

However, often it is impossible to verify the validity of these calculations. Using our system, it is possible to generate particles of known compositions and structures and to retrieve their refractive index, which can be compared to calculations by different mixing rules. We employ a variety of mixing rules to calculate the complex index of refraction for mixed aerosol and compare the calculated index with the index retrieved from our measurements.

We start with two non-absorbing components, $\mathrm{NaCl}$ and GA. The refractive indices retrieved for pure $\mathrm{NaCl}$ and $\mathrm{GA}$ aerosols are used as input for calculating the refractive indices of the mixtures by the different mixing rules and models. In all cases, $\mathrm{NaCl}$ is treated as the matrix and $\mathrm{GA}$ as the inclusion. Two delicate points are noted regarding the implementation of the mixing rules. First, mixing rules (2-4) require the volume fraction of the inclusion. When we calculate this volume fraction in a manner similar to that done in climate models (using the mass fraction and density of each substance), the volume fraction comes out too high. So instead, we calculate the volume fraction using the volumes of the solutions of $\mathrm{NaCl}$ and $\mathrm{GA}$ used to create each mixture. Second, rule (1) (molar refraction/absorption) requires the total molar volume of the mixture, defined as the total molecular weight divided by the total density, the latter of which is both difficult to measure and difficult to estimate if not dealing with tabulated solutions of electrolytes in water (e.g., Tang, 1997). Consequently, we make a similar assumption to Jacobson (2002) in such circumstances and calculate the total molar volume as: $V_{\mathrm{tot}}=\chi_{\mathrm{NaCl}}\left(\frac{M_{\mathrm{NaCl}}}{\rho_{\mathrm{NaCl}}}\right)+\chi_{\mathrm{GA}}\left(\frac{M_{\mathrm{GA}}}{\rho_{\mathrm{GA}}}\right)$, where $\chi_{i}$ is the molar ratio (not to be confused with the similar 
Table 1. Refractive index retrievals using Mie theory for sodium chloride, glutaric acid, and mixtures of the two with molar ratios 1:1 and $2: 1$, respectively. The retrieval was performed in one case using all the experimental sizes and in the other case using a subset of sizes starting from $350 \mathrm{~nm}$. The best fit was determined by obtaining the smallest merit function $\left(\chi^{2} / N^{2}\right)$.

\begin{tabular}{lllll}
\hline & \multicolumn{2}{c}{ Retrieval using all sizes } & \multicolumn{2}{c}{ Retrieval using subset of sizes (from $350 \mathrm{~nm}$ ) } \\
Sample & Refractive index & $\chi^{2} / N^{2}$ & Refractive index & $\chi^{2} / N^{2}$ \\
\hline $\mathrm{NaCl}$ & $1.546+\mathrm{i} 0.003$ & 0.04 & $1.544+\mathrm{i} 0.000$ & 0.09 \\
$\mathrm{Glutaric}$ acid & $1.410+\mathrm{i} 0.000$ & 0.10 & $1.410+\mathrm{i} 0.000$ & 0.13 \\
$1: 1 \mathrm{NaCl}$ : Glutaric acid & $1.483+\mathrm{i} 0.010$ & 0.06 & $1.480+\mathrm{i} 0.004$ & 0.08 \\
$2: 1 \mathrm{NaCl}$ : Glutaric acid & $1.507+\mathrm{i} 0.017$ & 0.01 & $1.507+\mathrm{i} 0.019$ & 0.02 \\
\hline
\end{tabular}
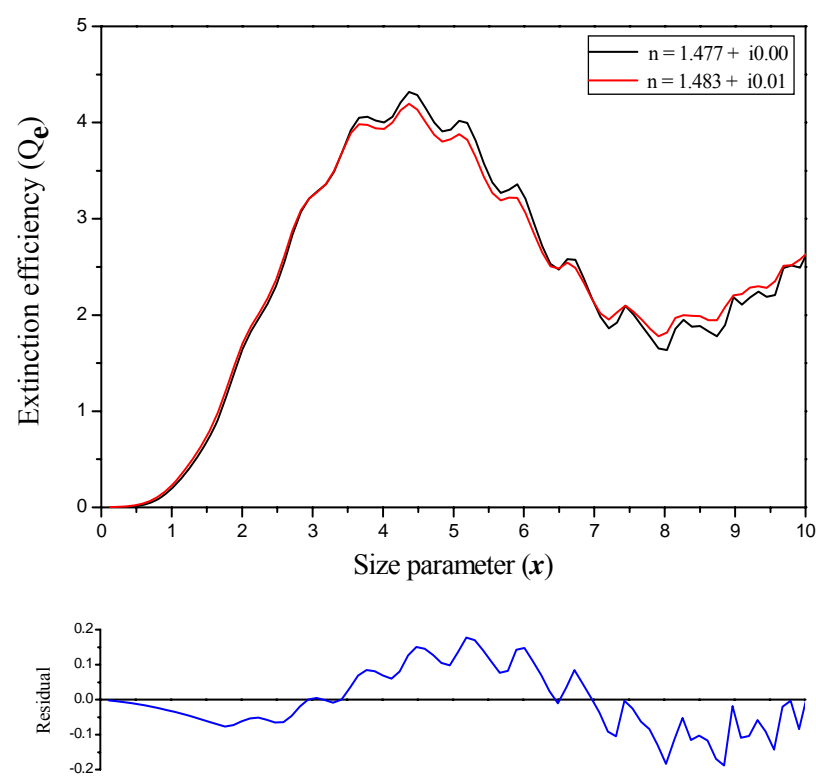

Fig. 7. Comparison between the refractive index obtained using linear mixing rule for the $1: 1$ mixture of $\mathrm{NaCl}$ and glutaric acid $(n=1.477+i 0.0)$ and the refractive index retrieved from measurements of the same mixture using Mie theory $(n=1.483+i 0.01)$. The curves are very similar for small size parameters, while differences are observed for higher size parameters. Note the different scale for the residuals.

variable in the merit function), $M_{i}$ is the molecular weight, and $\rho_{i}$ is the density (as tabulated for the substance's natural state).

Results of these calculations for 1:1 and 2:1 mixtures of $\mathrm{NaCl}$ and GA are given in Tables 2 and 3. The calculations are done for all the sizes and for the subset starting from $350 \mathrm{~nm}$ as indicated in the tables. Mixing rules (2-4) result in good agreement with the measurements. The smallest merit function value $(0.14)$ is obtained with the linear mixing rule, although the Maxwell-Garnett and EEMA also provide small merit function values and may also be appropriate for use in models. The molar refraction/absorption rule does not do as well, perhaps because our method for calculating the total molar volume is not accurate enough for these mixtures. The core plus shell model also produces a higher merit function than mixing rules (2-4), as might be expected from the fact that the $\mathrm{NaCl}$ and $\mathrm{GA}$ are homogeneously mixed in solution, not layered.

The refractive indices obtained by using mixing rules (24) are very close to those retrieved from the experimental data for both mixtures. For example, the linear mixing rule for all the sizes of the 1:1 mixture produce a refractive index of $n=1.477+\mathrm{i} 0.0$, while retrieved refractive index is $n=1.483+i 0.01$. A comparison between the extinction efficiency as a function of the size parameter of these two refractive indices and the residual between the curves are shown in Fig. 7. The curves are very close; differences in the curves appear at larger size parameters than were measured. We could probably improve the fit by measuring larger sizes, but the sizes we chose are more relevant to actual particles in the atmosphere.

3.5 Rhodamine 590, ammonium sulphate, and their mixtures

We used Rhodamine 590 (Rh-590) which has peak absorption in the visible around $530 \mathrm{~nm}$ as a model for strongly absorbing aerosols. In addition, we measure the optical properties of aerosols composed of mixtures of Rhodamine 590 and ammonium sulphate in four different molar ratios 1:10, 1:50, 1:100, and 1:500. Generating these aerosol mixtures is not as easy as the mixtures of $\mathrm{NaCl}$ and GA, since Rh-590 has a very low solubility in water $(0.1-1 \%)$, which could potentially affect the homogeneity of the aerosols during the atomizing process. To minimize this effect, we dissolve the $\mathrm{Rh}$ 590 in a $10 \%$ methanol/water solution. Because methanol is a fairly optically neutral substance (with real index close to that of water - between that of ammonium sulphate and Rh590 - and zero imaginary index), the possibility of a slight amount of methanol remaining in the aerosol is not likely to have altered the total extinction coefficient of the mixed aerosols by a measurable amount.

To retrieve the complex refractive index, we used the subroutine described in Sect. 2.3 for all the measured sizes and 
Table 2. The index of refraction of the mixture of $\mathrm{NaCl}$ and glutaric acid with molar ratio 1:1 obtained by using different mixing rules.

\begin{tabular}{|c|c|c|c|c|}
\hline \multicolumn{5}{|c|}{$\mathrm{NaCl}$ : Glutaric acid 1:1 (retrieved refractive index $1.483+\mathrm{i} 0.010$ and $\chi^{2} / N^{2}=0.06$ ) } \\
\hline \multirow[b]{2}{*}{ Mixing rule } & \multicolumn{2}{|c|}{ All experimental sizes } & \multicolumn{2}{|c|}{ Subset from $350 \mathrm{~nm}$} \\
\hline & Effective Refractive index & $\chi^{2} / N^{2}$ & Effective Refractive index & $\chi^{2} / N^{2}$ \\
\hline Molar refraction/absorption & $1.439+\mathrm{i} 0.000$ & 2.65 & $1.439+\mathrm{i} 0.000$ & 4.88 \\
\hline Linear & $1.477+\mathrm{i} 0.000$ & 0.14 & $1.477+\mathrm{i} 0.000$ & 0.14 \\
\hline Maxwell-Garnett & $1.477+\mathrm{i} 0.000$ & 0.15 & $1.477+\mathrm{i} 0.000$ & 0.15 \\
\hline EEMA, $d_{\text {incl }} \quad \S_{=0.01 \mu \mathrm{m}}$ & $1.475+\mathrm{i} 0.000$ & 0.18 & $1.475+\mathrm{i} 0.000$ & 0.21 \\
\hline $\mathrm{EEMA}, d_{\mathrm{incl}}=0.02 \mu \mathrm{m}$ & $1.477+\mathrm{i} 0.000$ & 0.18 & $1.475+\mathrm{i} 0.000$ & 0.21 \\
\hline $\mathrm{EEMA}, d_{\mathrm{incl}}=0.1 \mu \mathrm{m}$ & $1.476+\mathrm{i} 0.001$ & 0.15 & $1.476+\mathrm{i} 0.001$ & 0.16 \\
\hline
\end{tabular}

$\S$ EEMA $=$ extended effective medium approximation; $d_{\text {incl }}$ is the diameter of the inclusions assumed in the EEMA.

* There are no effective refractive indices in the core plus shell model. Separate refractive indices of the core and shell material are used.

Table 3. The index of refraction of the mixture of $\mathrm{NaCl}$ and glutaric acid with molar ratio 2:1 obtained by using different mixing rules.

\begin{tabular}{|c|c|c|c|c|}
\hline \multicolumn{5}{|c|}{$\mathrm{NaCl}$ : Glutaric acid 2:1 (retrieved refractive index $1.507+\mathrm{i} 0.017$ and $\left.\chi^{2} / N^{2}=0.01\right)$} \\
\hline \multirow[b]{2}{*}{ Mixing rule } & \multicolumn{2}{|c|}{ All experimental sizes } & \multicolumn{2}{|c|}{ Subset from $350 \mathrm{~nm}$} \\
\hline & Effective Refractive index & $\chi^{2} / N^{2}$ & Effective Refractive index & $\chi^{2} / N^{2}$ \\
\hline Molar refraction/absorption & $1.457+\mathrm{i} 0.000$ & 2.80 & $1.457+\mathrm{i} 0.000$ & 6.94 \\
\hline Linear & $1.499+\mathrm{i} 0.000$ & 0.14 & $1.499+\mathrm{i} 0.000$ & 0.23 \\
\hline Maxwell-Garnett & $1.499+\mathrm{i} 0.000$ & 0.15 & $1.499+\mathrm{i} 0.000$ & 0.25 \\
\hline $\mathrm{EEMA}, d_{\mathrm{incl}}=0.01 \mu \mathrm{m}$ & $1.498+\mathrm{i} 0.000$ & 0.17 & $1.498+\mathrm{i} 0.000$ & 0.29 \\
\hline $\mathrm{EEMA}, d_{\mathrm{incl}}=0.02 \mu \mathrm{m}$ & $1.498+\mathrm{i} 0.000$ & 0.17 & $1.498+\mathrm{i} 0.000$ & 0.29 \\
\hline $\mathrm{EEMA}, d_{\mathrm{incl}}=0.1 \mu \mathrm{m}$ & $1.499+\mathrm{i} 0.000$ & 0.14 & $1.499+\mathrm{i} 0.000$ & 0.24 \\
\hline
\end{tabular}

for a subset of sizes staring from $350 \mathrm{~nm}$. The extinction efficiency as a function of the size parameter for Rh-590, $\left(\mathrm{NH}_{4}\right)_{2} \mathrm{SO}_{4}$, and the different mixtures of the two are presented in Fig. 8a. The solid lines represent the refractive index retrieval using Mie theory with all of the measured sizes. The inset details the different aerosol compositions and the resulting complex refractive indices retrieved for each aerosol sample. As expected, as the fraction of absorbing material in the mixture decreases, the imaginary part of the refractive index decreases, and the real part of the complex refractive index increases.

Note that the retrieved refractive index for pure Rh-590 $(n=1.00+i 1.026)$ has a very low real part (close to that of air) and a high imaginary part. This can be expected from the fact that the wavelength of measurement is extremely close to the wavelength of peak absorption of Rh-590 (530 nm), so that we are essentially measuring at a frequency just below the resonance frequency. From the model of a dispersing medium attributed to H. A. Lorentz (Born and Wolf, 1999), we expect a corresponding peak in the imaginary part of the index and a value a little higher than 1.0 in the real part of the index. (Compare with the solid curve in Fig. 3 of Bulatov et al., 2006).

The extinction efficiency of the same aerosols, but with the refractive index retrieval algorithm applied only to the sub- set of sizes from $350 \mathrm{~nm}$ (solid curves) is shown in Fig. 8b. The fit in the small sizes region $(100-350 \mathrm{~nm})$ is as good as the fit in Fig. 8a, but in the region of larger sizes it is better. The retrieval shown in Fig. $8 b$ leads to a drastic change in the complex refractive index for the 1:10 mixture ratio, in which we obtained $n=1.203+\mathrm{i} 0.728$ in the retrieval using all the sizes and $n=1.405+\mathrm{i} 0.486$ in the retrieval using the subset of sizes. Likewise, the merit function for 1:10 mixture using the subset of sizes is much lower $\left(\chi^{2} / N^{2}=0.07\right)$ than that using all sizes $\left(\chi^{2} / N^{2}=0.3\right)$ (Table 4$)$. For the other mixtures, the differences between the two retrievals are not as drastic, as can be seen in the inset patterns in Figs. 8a and b and in Table 4, although the merit function using the subset of sizes is again generally lower than using all sizes. This could be explained by the presence of large, multiply charged particles.

\subsection{Calculations using mixing rules}

As in Sect. 3.4, we next employ a variety of mixing rules to calculate the complex index of refraction for the mixed aerosols and compare it with the index retrieved from our measurements in Sect. 3.3. The refractive indices retrieved for pure ammonium sulphate $(\mathrm{n}=1.52+\mathrm{i} 0.00)$ and pure $\mathrm{Rh}$ $590(n=1.00+11.026)$ are used as input for calculating the 
Table 4. The refractive indices of Rhodamine-590 (Rh-590) and mixtures of Rh-590 and ammonium sulphate with molar ratios 1:10, 1:50, 1:100, and 1:500, respectively obtained using the retrieval algorithm described in Sect. 2.3 to fit Mie theory with the experimental data. The fits were performed for two different size ranges. The first is for all the experimental sizes and the second using a subset sizes starting from $350 \mathrm{~nm}$. The best fit was determined by obtaining the smallest merit function $\left(\chi^{2} / N^{2}\right)$.

\begin{tabular}{lllll}
\hline & \multicolumn{2}{c}{ Retrieval using all sizes } & \multicolumn{2}{c}{ Retrieval using subset of sizes (from 350 nm) } \\
Sample & Refractive index & $\chi^{2} / N^{2}$ & Refractive index & $\chi^{2} / N^{2}$ \\
\hline $\mathrm{Rh}-590$ & $1.1+\mathrm{i} 1.16$ & 0.53 & $1.0+\mathrm{i} 1.03$ & 0.06 \\
$1: 10 \mathrm{Rh}-590:\left(\mathrm{NH}_{4}\right)_{2} \mathrm{SO}_{4}$ & $1.203+\mathrm{i} 0.728$ & 0.30 & $1.405+\mathrm{i} 0.486$ & 0.07 \\
$1: 50 \mathrm{Rh}-590:\left(\mathrm{NH}_{4}\right)_{2} \mathrm{SO}_{4}$ & $1.491+\mathrm{i} 0.462$ & 0.02 & $1.503+\mathrm{i} 0.420$ & 0.02 \\
$1: 100 \mathrm{Rh}-590:\left(\mathrm{NH}_{4}\right)_{2} \mathrm{SO}_{4}$ & $1.514+\mathrm{i} 0.291$ & 0.10 & $1.517+\mathrm{i} 0.236$ & 0.02 \\
$1: 500 \mathrm{Rh}-590:\left(\mathrm{NH}_{4}\right)_{2} \mathrm{SO}_{4}$ & $1.537+\mathrm{i} 0.132$ & 0.18 & $1.526+\mathrm{i} 0.103$ & 0.14 \\
\hline
\end{tabular}

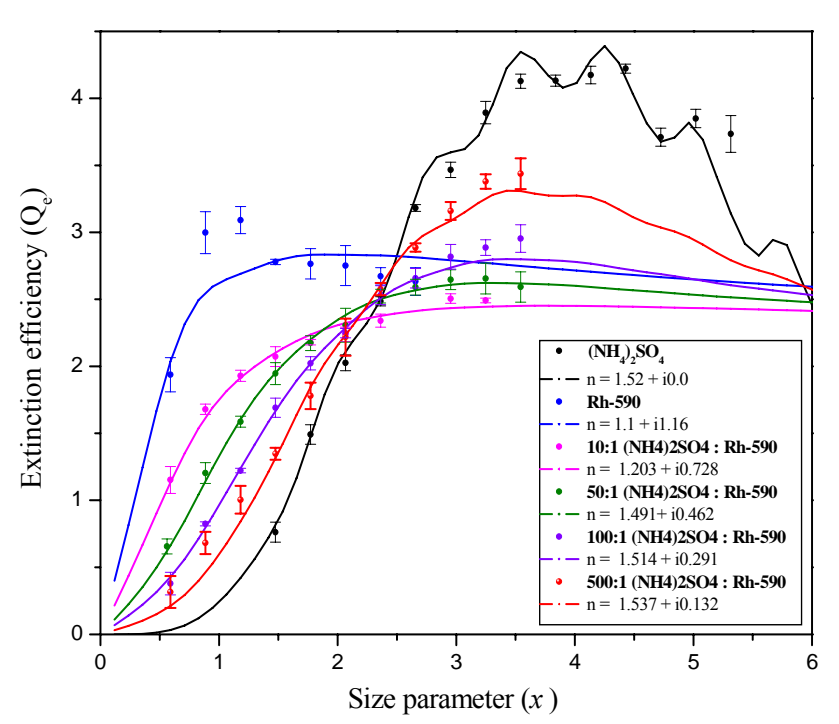

Fig. 8a. Extinction efficiency ( $\left.Q_{\text {ext }}\right)$ as a function of size parameter $(x)$ obtained for ammonium sulphate, Rhodamine 590 (Rh-590), and mixtures of the two with molar ratios 10:1, 50:1, 100:1, and $500: 1$, respectively. The standard deviation of the extinction efficiency for all of the experimental data is also presented. The solid lines represent the Mie theory fit for the corresponding aerosol samples using all experimental sizes.

refractive indices of the mixtures by the different mixing rules and models. In all cases, AS is treated as the matrix and $\mathrm{Rh}-590$ as the inclusion. As in the case of the $\mathrm{NaCl} / \mathrm{GA}$ mixtures, the volume fraction of the inclusion is calculated using the volumes of the solutions of AS and Rh-590 used to create the mixture. Unlike the case of the $\mathrm{NaCl} / \mathrm{GA}$ mixtures, we have less information regarding the proper molecular weight and density of Rh-590 (in solid or liquid phase), so we do not include the molar refraction/absorption mixing rule here.

Results of the calculations using different mixing rules for the 1:10, 1:50, and 1:100 mixture sample are given in Tables 5, 6, and 7. The calculations are performed for all sizes and for a subset of sizes starting from $350 \mathrm{~nm}$ as indicated in

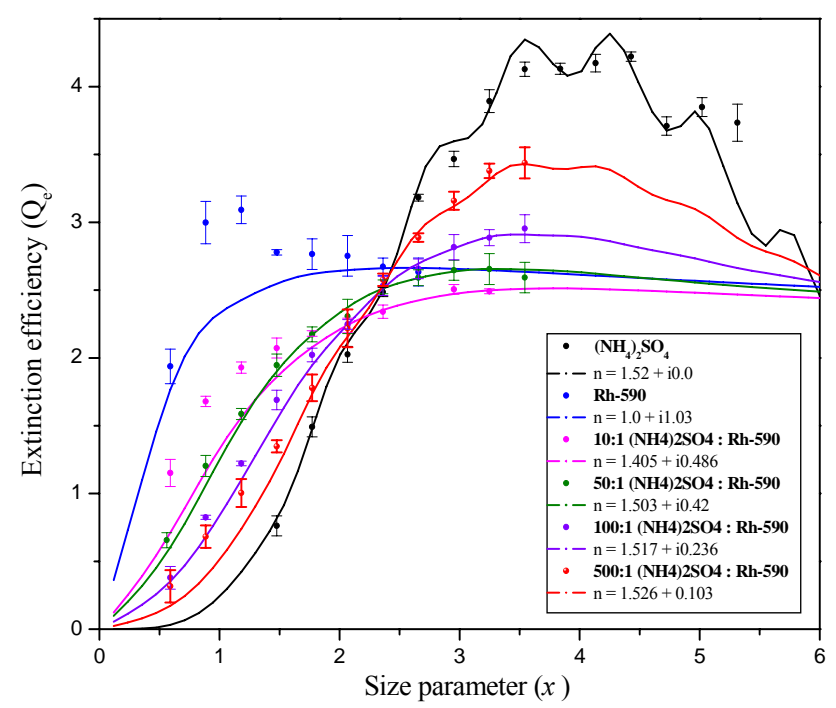

Fig. 8b. Extinction efficiency $\left(Q_{\text {ext }}\right)$ as a function of the size parameter $(x)$ obtained for ammonium sulphate, Rhodamine 590 (Rh590), and mixtures of the two with molar ratios 10:1, 50:1, 100:1, and 500:1, respectively. The standard deviation of the extinction efficiency for all of the experimental data is also presented. The solid lines represent the Mie theory fit for the corresponding aerosol sample. The solid lines represent the Mie theory fit for the corresponding aerosol samples using a subset of experimental sizes starting from $350 \mathrm{~nm}$.

the table. Calculations performed on the subset of sizes give a considerably small merit function comparing to the calculations performed on all sizes, up to an order of magnitude smaller. In addition, although the AS and Rh590 are homogeneously mixed in solution in a fashion similar to the mixtures of $\mathrm{NaCl}$ and $\mathrm{GA}$, the mixing rule providing the smallest merit function is the extended effective medium approximation assuming inclusions of radius $d_{\text {incl }}=0.01 \mu \mathrm{m}$. This indicates that accounting for absorption in a mixture is better achieved with a higher order mixing rule (higher order in inclusion size) than with a lower or zeroith order mixing 
Table 5. The index of refraction obtained for the mixture of Rh-590 and ammonium sulphate with molar ratio 1:10 obtained using different mixing rules.

\begin{tabular}{|c|c|c|c|c|}
\hline \multicolumn{5}{|c|}{$\mathrm{Rh}-590:\left(\mathrm{NH}_{4}\right)_{2} \mathrm{SO}_{4} 1: 10$ (retrieved refractive index $1.203+\mathrm{i} 0.728$ and $\left.\chi^{2} / N^{2}=0.30\right)$} \\
\hline \multirow[b]{2}{*}{ Mixing rule } & \multicolumn{2}{|c|}{ All experimental sizes } & \multicolumn{2}{|c|}{ Subset from $350 \mathrm{~nm}$} \\
\hline & Effective refractive index & $\chi^{2} / N^{2}$ & Effective refractive index & $\chi^{2} / N^{2}$ \\
\hline Linear & $1.416+\mathrm{i} 0.203$ & 33.77 & $1.416+\mathrm{i} 0.205$ & 7.13 \\
\hline Maxwell-Garnett & $1.397+\mathrm{i} 0.251$ & 27.82 & $1.397+\mathrm{i} 0.251$ & 5.85 \\
\hline EEMA, $d_{\text {incl }}=0.01 \mu \mathrm{m}$ & $1.422+\mathrm{i} 0.276$ & 20.89 & $1.422+\mathrm{i} 0.276$ & 2.95 \\
\hline EEMA, $d_{\text {incl }}=0.02 \mu \mathrm{m}$ & $1.421+\mathrm{i} 0.274$ & 21.23 & $1.421+\mathrm{i} 0.274$ & 3.01 \\
\hline $\mathrm{EEMA}, d_{\mathrm{incl}}=0.1 \mu \mathrm{m}$ & $1.392+\mathrm{i} 0.210$ & 36.93 & $1.392+\mathrm{i} 0.210$ & 9.57 \\
\hline
\end{tabular}

Table 6. The index of refraction obtained for the mixture of Rh-590 and ammonium sulphate with molar ratio 1:50 obtained using different mixing rules.

\begin{tabular}{llrlr}
\hline \multicolumn{2}{c}{$\begin{array}{c}\mathrm{Rh}-590:\left(\mathrm{NH}_{4}\right)_{2} \mathrm{SO}_{4} \\
\text { 1:50 (retrieved refractive index } \\
\text { Mixing rule }\end{array}$} & $\begin{array}{c}\left.1.491+\mathrm{i} 0.462 \text { and } \chi^{2} / N^{2}=0.02\right) \\
\text { All experimental sizes }\end{array}$ \\
& Effective refractive index & $\chi^{2} / N^{2}$ & Effective refractive index & $\chi^{2} / N^{2}$ \\
\hline Linear & $1.481+\mathrm{i} 0.076$ & 10.47 & $1.481+\mathrm{i} 0.076$ & 3.63 \\
Maxwell-Granett & $1.475+\mathrm{i} 0.095$ & 9.38 & $1.475+\mathrm{i} 0.095$ & 2.83 \\
EEMA, $d_{\text {incl }}=0.01 \mu \mathrm{m}$ & $1.478+\mathrm{i} 0.098$ & 9.03 & $1.478+\mathrm{i} 0.098$ & 2.71 \\
EEMA, $d_{\text {incl }}=0.02 \mu \mathrm{m}$ & $1.478+\mathrm{i} 0.098$ & 9.08 & $1.478+\mathrm{i} 0.098$ & 2.73 \\
EEMA, $d_{\text {incl }}=0.1 \mu \mathrm{m}$ & $1.470+\mathrm{i} 0.074$ & 11.10 & $1.470+\mathrm{i} 0.074$ & 3.67 \\
\hline
\end{tabular}

rule, although the size of the inclusions assumed may need to remain small to better simulate the homogeneity of the mixture. As with the non-absorbing mixtures, the core plus shell model tends to produce a higher merit function for the $1: 10$, 1:50, and 1:100 Rh590-AS mixtures and is less appropriate. Results of the calculations using different mixing rules for the 1:500 Rh590-AS mixture samples are given in Table 8. For this dilute mixture, even though Rh590 is highly absorbing, most mixing rules and models give similar merit function values, although the linear model provides the highest (worst) merit function, and interestingly the core plus shell model provides the lowest (best). The mixing rules give similar merit functions because they are of similar applicability for dilute inclusions. (One of the assumptions implicit in mixing rules is that of "dilute suspensions".)

Having said all that, it should be noted that no mixing rule or model provides a very low merit function in the case of the absorbing mixtures; all merit function values are of an order of magnitude higher than the merit functions provided by the refractive index retrievals. Furthermore, there is a better agreement in the real part of the refractive index, while there is less agreement in the imaginary part. So we can choose the best mixing rule or model, but none will give an excellent match to the measurements at the volume fractions of Rh590 tested here, as opposed to the application of the mixing rules on the non-absorbing mixtures in Sect. 3.4 which give a better match.

\section{Summary and outlook}

We applied cavity ring down measurements of the optical properties of absorbing and non-absorbing aerosols and their different mixtures. We performed a few modifications in the way the aerosols enter and exit the cavity enabling a uniform distribution of the aerosols inside the cavity, which leads to more accurate measurements of the extinction coefficient. The system was validated by measuring the extinction coefficient, extinction efficiency, and the extinction cross section and refractive indices of size selected polystyrene spheres and ammonium sulphate aerosols. The refractive indices for these aerosols were retrieved from the measurements using Mie theory and are in good agreement with data in the literature.

The thrust of this study was the use of the CRD technique to determine the optical properties of aerosols composed of mixtures of different absorbing and non-absorbing species and to determine their complex refractive indices. In addition, we were interested in applying different mixing rules used for calculations of refractive indices and comparing them with the measurements. For non-absorbing mixtures of sodium chloride and glutaric acid, we found very good agreement between the measurements and calculated values. Among the different mixing rules and models, the linear mixing rule provided the smallest merit function, suggesting that for these non-absorbing species, the linear mixing rule 
Table 7. The index of refraction obtained for the mixture of Rh-590 and ammonium sulphate with molar ratio 1:100 obtained using different mixing rules.

\begin{tabular}{|c|c|c|c|c|}
\hline \multicolumn{5}{|c|}{ Rh-590: $\left(\mathrm{NH}_{4}\right)_{2} \mathrm{SO}_{4} 1: 100$ (retrieved refractive index $1.514+\mathrm{i} 0.291$ and $\left.\chi^{2} / N^{2}=0.10\right)$} \\
\hline \multirow[b]{2}{*}{ Mixing rule } & \multicolumn{2}{|c|}{ All experimental sizes } & \multicolumn{2}{|c|}{ Subset from $350 \mathrm{~nm}$} \\
\hline & Effective refractive index & $\chi^{2} / N^{2}$ & Effective refractive index & $\chi^{2} / N^{2}$ \\
\hline Linear & $1.500+\mathrm{i} 0.040$ & 31.26 & $1.500+\mathrm{i} 0.040$ & 6.99 \\
\hline Maxwell-Garnett & $1.497+\mathrm{i} 0.050$ & 28.87 & $1.497+\mathrm{i} 0.050$ & 6.00 \\
\hline EEMA, $d_{\text {incl }}=0.01 \mu \mathrm{m}$ & $1.498+\mathrm{i} 0.050$ & 28.55 & $1.498+\mathrm{i} 0.050$ & 5.92 \\
\hline EEMA, $d_{\text {incl }}=0.02 \mu \mathrm{m}$ & $1.497+\mathrm{i} 0.050$ & 28.65 & $1.497+\mathrm{i} 0.050$ & 5.95 \\
\hline $\mathrm{EEMA}, d_{\mathrm{incl}}=0.1 \mu \mathrm{m}$ & $1.494+\mathrm{i} 0.038$ & 32.47 & $1.494+\mathrm{i} 0.038$ & 7.11 \\
\hline
\end{tabular}

Table 8. The index of refraction obtained for the mixture of Rh-590 and ammonium sulphate with molar ratio 1:500 obtained using different mixing rules.

\begin{tabular}{|c|c|c|c|c|}
\hline \multicolumn{5}{|c|}{$\mathrm{Rh}-590:\left(\mathrm{NH}_{4}\right)_{2} \mathrm{SO}_{4} 1: 500$ (retrieved refractive index $1.537+\mathrm{i} 0.132$ and $\left.\chi^{2} / N^{2}=0.18\right)$} \\
\hline \multirow[b]{2}{*}{ Mixing rule } & \multicolumn{2}{|c|}{ All experimental sizes } & \multicolumn{2}{|c|}{ Subset start from $350 \mathrm{~nm}$} \\
\hline & Effective Refractive index & $\chi^{2} / N^{2}$ & Effective Refractive index & $\chi^{2} / N^{2}$ \\
\hline Linear & $1.516+\mathrm{i} 0.008$ & 3.23 & $1.516+\mathrm{i} 0.008$ & 4.26 \\
\hline Maxwell-Granett & $1.515+\mathrm{i} 0.013$ & 3.08 & $1.515+\mathrm{i} 0.013$ & 3.90 \\
\hline EEMA, $d_{\text {incl }}=0.01 \mu \mathrm{m}$ & $1.515+\mathrm{i} 0.010$ & 3.08 & $1.515+\mathrm{i} 0.010$ & 3.90 \\
\hline EEMA, $d_{\text {incl }}=0.02 \mu \mathrm{m}$ & $1.515+\mathrm{i} 0.010$ & 3.08 & $1.515+\mathrm{i} 0.010$ & 3.90 \\
\hline $\mathrm{EEMA}, d_{\mathrm{incl}}=0.1 \mu \mathrm{m}$ & $1.515+\mathrm{i} 0.008$ & 3.22 & $1.515+\mathrm{i} 0.008$ & 4.09 \\
\hline
\end{tabular}

would be the best to predict the index of refraction. However, other mixing rules also provide very good agreement with the measured values. To the best of our knowledge, this is the first attempt to use CRD spectroscopy to test the performance of mixing rules in determining the index of refraction of aerosols with known composition and mixture ratio.

Similarly, for absorbing mixtures with low volume fractions of the absorbing substance, all mixing rules provide similar results, with the core plus shell model providing slightly better results than the others. For absorbing mixtures with relatively high volume fractions of the absorbing substance, no mixing rule or model provides an excellent match to measurements, although an extended effective medium approximation (higher order in the size of the inclusions) provides a lower merit function in comparison to measurements than the others.

The recent systematic increase in the uses of cavity ring down spectroscopy for studying optical properties of aerosols justify the advantages of this technique, and certainly further modifications in this technique would make it more suitable for measurements of different types of aerosols in both field and laboratory measurements. Many more configurations of aerosol mixtures can be studied, including off-resonance absorption and coated particles, both absorbing and nonabsorbing.
Acknowledgements. The authors acknowledge the help given by I. Schechter and V. Bultov with the new CRD setup. The help of C. Spindler is highly appreciated. Financial support for this work was provided by the Minerva foundation of the Max Planck society and by Intel Inc. C. Erlick acknowledges support from the Israel Science Foundation grant 1315/04.

Edited by: C. George

\section{References}

Bates, T. S., Anderson, T. L., Baynard, T., Bond, T., Boucher, O., Carmichael, G., Clarke, A., Erlick, C., Guo, H., Horowitz, L., Howell, S., Kulkarni, S., Maring, H., McComiskey, A., Middlebrook, A., Noone, K., O’Dowd, C. D., Ogren, J., Penner, J., Quinn, P. K., Ravishankara, A. R., Savoie, D. L., Schwartz, S. E., Shinozuka, Y., Tang, Y., Weber, R. J., and Wu, Y.: Aerosol direct radiative effects over the northwest atlantic, northwest pacific, and north indian oceans: Estimates based on in-situ chemical and optical measurements and chemical transport modeling, Atmos. Chem. Phys., 6, 1657-1732, 2006, http://www.atmos-chem-phys.net/6/1657/2006/.

Bellouin, N., Boucher, O., Haywood, J., and Reddy, M. S.: Global estimate of aerosol direct radiative forcing from satellite measurements, Nature, 438, 1138-1141, 2005.

Bohren, C. F. and Huffman, D. R.: Absorption and scattering of light by small particles, Wiley, New York, 1983. 
Bohren, C. F. and Clothiaux, E. E.: Fundamentals of atmospheric radiation, Wiley-VCH, 2006.

Born, M. and Wolf, E.: Principles of optics, 7th ed., Cambridge University Press, 1999.

Brown, S. S., Stark, H., Ciciora, S. J., McLaughlin, R. J., and Ravishankara, A. R.: Simultaneous in situ detection of atmospheric no3 and n2o5 via cavity ring-down spectroscopy, Rev. Sci. Inst., 73, 3291-3301, 2002.

Bulatov, V., Chen, Y. H., Khalmanov, A., and Schechter, I.: Absorption and scattering characterization of airborne microparticulates by a cavity ringdown technique, Anal. Bioanal. Chem., 384, 155160, 2006.

Bulatov, V., Fisher, M., and Schechter, I.: Aerosol analysis by cavity-ring-down laser spectroscopy, Analytica Chimica Acta, 466, 1-9, 2002.

Chýlek, P., Videen, G., Geldart, D. J. W., Dobbie, J. S., and Tso, H. C. W.: Effective medium approximations for heterogeneous particles, in: Light scattering by nonspherical particles, theory, measurements, and applications, pp. 273-308, edited by: Mishchenko, M. I., Hovenier, J. W., and Travis, L. D., Academic Press, 2000.

Chylek, P., Ramaswamy, V., and Cheng, R. J.: Effect of graphitic carbon on the albedo of clouds, J. Atmos. Sci., 41, 3076-3084, 1984.

d'Almeida, G. A., Koepke, P., and Shettle, E. P.: Atmospheric aerosols, global climatology and radiative characteristics, A. Deepak Publishing, Hampton, Va, 1991.

Erlick, C.: Effective refractive indices of water and sulfate drops containing absorbing inclusions, J. Atmos. Sci., 63, 754-763, 2006.

Falkovich, A. H., Schkolnik, G., Ganor, E., and Rudich, Y.: Adsorption of organic compounds pertinent to urban environments onto mineral dust particles, J. Geophys. Res., 109, D02208, doi:10.1029/2003JD003919, 2004.

Gosse, S. F., Wang, M. Y., Labrie, D., and Chylek, P.: Imaginary part of the refractive index of sulfates and nitrates in the $0.7-$ 2.8 mum spectral region, Appl. Opt., 36, 3622-3634, 1997.

Hansen, J., Sato, M., Ruedy, R., Nazarenko, L., Lacis, A., Schmidt, G. A., Russell, G., Aleinov, I., Bauer, M., Bauer, S., Bell, N., Cairns, B., Canuto, V., Chandler, M., Cheng, Y., Del Genio, A., Faluvegi, G., Fleming, E., Friend, A., Hall, T., Jackman, C., Kelley, M., Kiang, N., Koch, D., Lean, J., Lerner, J., Lo, K., Menon, S., Miller, R., Minnis, P., Novakov, T., Oinas, V., Perlwitz, J., Perlwitz, J., Rind, D., Romanou, A., Shindell, D., Stone, P., Sun, S., Tausnev, N., Thresher, D., Wielicki, B., Wong, T., Yao, M., and Zhang, S.: Efficacy of climate forcings, J. Geophys. Res., 110, D18104, doi:10.1029/2005JD005776, 2005.

Jacobson, M. C.: Strong radiative heating due to the mixing state of black carbon in atmospheric aerosols, Nature, 409, 695-697, 2001.

Jacobson, M. Z.: Analysis of aerosol interactions with numerical techniques for solving coagulation, nucleation, condensation, dissolution, and reversible chemistry among multiple size distributions, J. Geophys. Res., 107(D19), 4366, doi:10.1029/2001JD002044, 2002.

Jacobson, M. Z.: Effects of externally-through-internally-mixed soot inclusions within clouds and precipitation on global climate, J. Phys. Chem. A, 110, 6860-6873, 2006.

Kanakidou, M., Seinfeld, J. H., Pandis, S. N., Barnes, I., Dentener,
F. J., Facchini, M. C., Van Dingenen, R., Ervens, B., Nenes, A., Nielsen, C. J., Swietlicki, E., Putaud, J. P., Balkanski, Y., Fuzzi, S., Horth, J., Moortgat, G. K., Winterhalter, R., Myhre, C. E. L., Tsigaridis, K., Vignati, E., Stephanou, E. G., and Wilson, J.: Organic aerosol and global climate modelling: A review, Atmos. Chem. Phys., 5, 1053-1123, 2005, http://www.atmos-chem-phys.net/5/1053/2005/.

Kaufman, Y. J., Koren, I., Remer, L. A., Rosenfeld, D., and Rudich, Y.: The effect of smoke, dust, and pollution aerosol on shallow cloud development over the atlantic ocean, Proc. Natl. Acad. Sci. USA, 102, 11 207-11 212, 2005.

Kaufman, Y. J., Tanre, D., and Boucher, O.: A satellite view of aerosols in the climate system, Nature, 419, 215-223, 2002.

Koren, I., Kaufman, Y. J., Remer, L. A., and Martins, J. V.: Measurement of the effect of amazon smoke on inhibition of cloud formation, Science, 303, 1342-1345, 2004.

Lack, D. A., Lovejoy, E. R., Baynard, T., Pettersson, A., and Ravishankara, A. R.: Aerosol absorption measurement using photoacoustic spectroscopy: Sensitivity, calibration, and uncertainty developments, Aerosol. Sci. Technol., 40, 697-708, 2006.

Lide, D. R.: Crc handbook of chemistry and physics, 78 ed., CRC Press LLC, Boca Raton, Florida, USA, 1997.

Lohmann, U. and Feichter, J.: Global indirect aerosol effects: A review, Atmos. Chem. Phys., 5, 715-737, 2005, http://www.atmos-chem-phys.net/5/715/2005/.

Maria, S. F., Russell, L. M., Gilles, M. K., and Myneni, S. C. B.: Organic aerosol growth mechanisms and their climate-forcing implications, Science, 306, 1921-1924, 2004.

Menon, S., Hansen, J., Nazarenko, L., and Luo, Y.: Climate effects of black carbon aerosols in china and india, Science, 297, 2250 2253, 2002.

Moosmuller, H., Varma, R., and Arnott, W. P.: Cavity ring-down and cavity-enhanced detection techniques for the measurement of aerosol extinction, Aero. Sci. Tech., 39, 30-39, 2005.

Murphy, D. M., Cziczo, D. J., Froyd, K. D., Hudson, P. K., Matthew, B. M., Middlebrook, A. M., Peltier, R. E., Sullivan, A., Thomson, D. S., and Weber, R. J.: Single-particle mass spectrometry of tropospheric aerosol particles, J. Geophys. Res., 111, D23S32, doi:10.1029/2006JD007340, 2006.

O'Keefe, A. and Deacon, D. A. G.: Cavity ring-down optical spectrometer for absorption-measurements using pulsed laser sources, Rev. Sci. Inst., 59, 2544-2551, 1988.

Pettersson, A., Lovejoy, E. R., Brock, C. A., Brown, S. S., and Ravishankara, A. R.: Measurement of aerosol optical extinction at $532 \mathrm{~nm}$ with pulsed cavity ring down spectroscopy, J. Aerosol Sci., 35, 995-1011, 2004.

Posfai, M., Xu, H. F., Anderson, J. R., and Buseck, P. R.: Wet and dry sizes of atmospheric aerosol particles: An afm-tem study, Geophys. Res. Lett., 25, 1907-1910, 1998.

Press, W. H., Teukolsky, S. A., Vetterling, W. T., and Flannery, B. R.: Numerical recipes in c, the art of scientific computer, 2nd edition, Cambridge University Press, New York, 1992.

Ramanathan, V., Chung, C., Kim, D., Bettge, T., Buja, L., Kiehl, J. T., Washington, W. M., Fu, Q., Sikka, D. R., and Wild, M.: Atmospheric brown clouds: Impacts on south asian climate and hydrological cycle, Proc. Natl. Acad. Sci. USA, 102, 5326-5333, 2005.

Ramanathan, V., Crutzen, P. J., Kiehl, J. T., and Rosenfeld, D.: Atmosphere - aerosols, climate, and the hydrological cycle, Sci- 
ence, 294, 2119-2124, 2001.

Russell, L. M., Maria, S. F., and Myneni, S. C. B.: Mapping organic coatings on atmospheric particles, Geophys. Res. Lett., 29, 16, doi:10.1029/2002GL014874, 2002.

Sappey, A. D., Hill, E. S., Settersten, T., and Linne, M. A.: Fixedfrequency cavity ringdown diagnostic for atmospheric particulate matter, Optics Lett., 23, 954-956, 1998.

Scherer, J. J., Paul, J. B., Collier, C. P., OKeefe, A., Rakestraw, D. J., and Saykally, R. J.: Cavity ringdown laser spectroscopy: A new ultrasensitive absorption technique, Spectroscopy, 11, 4650, 1996.

Scherer, J. J., Paul, J. B., OKeefe, A., and Saykally, R. J.: Cavity ringdown laser absorption spectroscopy: History, development, and application to pulsed molecular beams, Chem. Rev., 97, 25$51,1997$.

Sihvola, A. and Sharma, R.: Scattering corrections for maxwell garnett mixing rule, Microwave and Optical Technology Letters, 22, 229-231, 1999.

Smith, J. D. and Atkinson, D. B.: A portable pulsed cavity ringdown transmissometer for measurement of the optical extinction of the atmospheric aerosol, Analyst, 126, 1216-1220, 2001.

Stelson, A. W.: Urban aerosol refractive-index prediction by partial molar refraction approach, Environ. Sci. Technol., 24, 16761679, 1990.
Strawa, A. W., Castaneda, R., Owano, T., Baer, D. S., and Paldus, B. A.: The measurement of aerosol optical properties using continuous wave cavity ring-down techniques, J. Atmos. Oceanic Technol., 20, 454-465, 2003.

Tang, I. N.: Thermodynamic and optical properties of mixedsalt aerosols of atmospheric importance, J. Geophys. Res., 102, 1883-1893, 1997.

Tervahattu, H., Hartonen, K., Kerminen, V. M., Kupiainen, K., Aarnio, P., Koskentalo, T., Tuck, A. F., and Vaida, V.: New evidence of an organic layer on marine aerosols, J. Geophys. Res., 107, 4053, 2002.

Thompson, J. E., Smith, B. W., and Winefordner, J. D.: Monitoring atmospheric particulate matter through cavity ring-down spectroscopy, Anal. Chem., 74, 1962-1967, 2002.

Vander Wal, R. L. and Ticich, T. M.: Cavity ringdown and laserinduced incandescence measurements of soot, Appl. Opt., 38, 1444-1451, 1999.

Yu, H., Kaufman, Y. J., Chin, M., Feingold, G., Remer, L. A., Anderson, T. L., Balkanski, Y., Bellouin, N., Boucher, O., Christopher, S., DeCola, P., Kahn, R., Koch, D., Loeb, N., Reddy, M. S., Schulz, M., Takemura, T., and Zhou, M.: A review of measurement-based assessments of the aerosol direct radiative effect and forcing, Atmos. Chem. Phys., 6, 613-666, 2006, http://www.atmos-chem-phys.net/6/613/2006/. 